\title{
Research on the Construction of Sports Resources Information Platform Based on Big Data
}

\author{
Wang Shuangming ${ }^{1,2}$ \\ ${ }^{1 .}$ Malaysia University of Science and Technology \\ Kuala Lumpur, Malaysia \\ ${ }^{2}$ Dean's Office, Xi'an Siyuan University \\ Xi'an, China \\ e-mail: 646422452@qq.com \\ Shi Jianwei \\ Personnel office, Xi'an Siyuan University \\ Xi'an, China \\ e-mail: 95003882 @ qq.com
}

\author{
Fu Shiqiu \\ College of Liberal Arts \\ Xi'an Siyuan University \\ Xi'an, China \\ e-mail: 137792522 @qq.com
}

\author{
Xu Wanlin \\ Sports departmen \\ Xi'an Siyuan University \\ Xi'an, China \\ e-mail: $957294947 @$ @q.com
}

\begin{abstract}
The rapid development of communication technology such as big data makes the integration and application of sports resource information become the key of sports informatization construction. The construction of sports resources information big data platform is of great significance to the integration of sports resources and the sharing of sports resources. The subject explores the related theory of sports resources, studies the present situation of the application of sports resources, constructs the overall construction framework of sports resources information big data platform, analyzes and designs the functions of each subsystem, and discusses the performance evaluation of sports resources information big data platform.
\end{abstract}

Keywords-Big Data; Sports Resources Information; Platform Construction

\section{INTRODUCTION}

\section{A. The advent of big data era}

The State Council's "Outline for Accelerating the Development of Big Data" (GuoFa [2015] No. 50) states: The convergence of information technology and economic society has led to the rapid growth of data. The data have become a national basic strategic resource. Big data is increasingly being used globally, with an important impact on global production, circulation, distribution, consumption activities as well as economic operation mechanism, social life style and national governance ability.[1] At present, China ranks first in the world in the scale of Internet and mobile Internet users with abundant data resource and application market advantages. Breakthroughs have been made in key data technology research and development in some big data sectors. A number of Internet innovative enterprises and innovative applications have emerged. Some local governments have started big-data-related work. Insisting on innovation-driven development, accelerating the deployment of big data and deepening the application of big data have become the inherent and necessary choices of stabilizing growth, promoting reforms, adjusting structure, benefiting people's livelihood, and promoting the modernization of government governance.[2]Big data technology opens up a whole new era of opportunities and challenges.

Big data is a data set featuring large capacity, multiple types, high speed access and high application value. It is rapidly evolving into collecting, storing and associating and analyzing a large amount of data with diverse sources and diverse formats, which will discover new knowledge, create new values and upgrade new capabilities in a new generation of information technology and service forms. [3] Big data is featured by massive scale, high-speed circulation and rich forms, which has been widely used and developed in IT, aviation, e-commerce and other industries. Big data complements and improves traditional data collection methods, which expands the breadth and depth of data usage.

\section{B. The development of sports informatization}

The emergence of "Internet+" has promoted the innovation and development of information technology in the industry.[4] "National Fitness Program (2016-2020)" pointed out that we must promote depth integration between "Internet+", big data and other information technology and the National Fitness Program, so as to facilitate the construction of national fitness public service information platform.[5] The "13th Five-Year Plan for Sports Development" issued by General Administration of Sport of China affirmed that "Internet+" has infused new vitality into sports development and should make full use of new technologies to develop various types of APP to open up the customer market.[6] Under the guidance of the programmatic documents, sports informatization has made some progress. 


\section{Sports resources integration needs}

"The contradiction between the growing mass sports demand and the relative insufficiency of social sports resources "[7] has become increasingly acute. Relevant statistics show that the number of people who regularly participate in physical exercise in China has increased by $5.7 \%$ in the recent seven years, while that of sports supported by public finance has dropped by $0.13 \%$.

On the one hand, there are largely idle sports facilities which are heavily subsidized by the government for largescale sporting events. Most sports venues built by individuals or social groups are poorly equipped with few visitors, and the utilization rate of sports facilities in schools and enterprises is low; on the other hand, residents can not find a place suitable for exercise. The reasons are as follows: first, the distribution of public sports resources is not balanced; second, the dislocation of supply and demand of sports resources "aristocratic supply" and "civilian needs"; third, the information asymmetry of supply and demand of sports resources. Taking "Internet+" as an opportunity, we shall use big data technology to build a sports resource information platform to precisely match users and sports resources and improve the utilization rate of sports resources and satisfaction of all parties.

\section{OVERVIEW OF SPORTS RESOURCES BIG DATA}

\section{A. Sports informatization}

The ultimate goal of sports development is to better provide the public with satisfying sports products and services. The matching degree between sports informationization and public demand can most directly reflect the construction results of sports. Judging from the current situation of the development of sports informationization, there is still a big gap between sports information service and public demand, which is mainly manifested in the following three aspects:

1) Less capable of collaborative development:Sports information management agencies are always at a low level, we do not attach importance to the training and stability of the sports information personnel, with the lack of necessary follow-up financial security. Sports information construction is not highly motivated by the management level, operational level and the implementation level or with inconsistent understanding, and there is no system or mechanism to organize and coordinate the relationship among the government, sports departments and the masses, leading to the lack of sustainable development of sports information construction.

2) The level of information technology application is generally low:Due to the fragmentation of the application of sports information technology, the poor circulation of information resources and the low involvement of new technologies, the scientification degree of sports management, planning, development and decision-making is low. The success stories of sports information systems are few, also with the lack of key projects promoted by works in all areas.

3) Inefficient access to service information:The growth of service resources failed to meet the growing public demand and public satisfaction was poor. On the one hand, the release of service information is often limited to the news media, policy documents, government websites, etc. The coverage and transmission speed of information are affected to some extent, and the public can not obtain real-time, comprehensive and accurate service information through mobile terminals. On the other hand, there are too few valuable service functions available online, resulting in low public participation.

As for the specific sports resources big data, it also faces many challenges. The problems that need to be solved are as follows: The old and new software and hardware systems are difficult to integrate in depth, and the applications and data based on the system are featured by low relevance and poor sharing; Unscientific data classification and low data utilization; Large differences among data formats and serious data redundancy.

Sports resource information big data content is huge, which involves a large number of departments, so we shall properly solve the above problems and make the sports resources information database to better serve the public.

\section{B. Concept of Sports Resource Information Platform Based on Big Data}

The information technology is used to mine the information contained in the big data of sports resources and store the information according to certain rules, so that a massive sports information database is constructed, and a multi-user information platform is constructed based on the sports resources information database. Such a platform is called sports resources information big data platform.

\section{ANALYSIS AND DESIGN OF SPORTS RESOURCE INFORMATION PLATFORM BASED ON BIG DATA}

\section{A. Construction Principles of Sports Resource Information Platform Based on Big Data}

The construction principles of Sports Resource Information Platform Based on Big Data include:

1) Unity:The development of a unified technical specifications. the construction of the sports resources information platform based on big data must adopt a unified technology architecture, so as to ensure the smooth implementation of the system and good application results.

2) Security: The platform security is the lifeblood of the platform, so we should attach great importance to platform application security, data security and overall security in the platform implementation process. Upholding the principle of reliability, we shall to the maximum extent possible avoid business failures due to technical failure.

3) Reliability: The platform should use mature technologies and interfaces to make full use of the existing 
sports resource applications to avoid the construction risk and the use risk of the platform due to technical defects.

4) Scalability: The sports resource information platform based on big data should be designed and developed based on the needs of the sports management department, sports resource providers and sports resource users, and should give full consideration to the adaptability of the platform to the business changes of all stakeholders. The platform design and development may reduce the impact of business changes on the platform and achieve stable support for business changes through proper expansion and simple modification of the platform.

5) Forward-looking: The platform construction should be forward-looking, and we must consider the needs of business updates in the next few years, learn from the advanced international and domestic experience, and adopt the successful technology of information platform construction so that the platform can reflect the advanced nature in the future.

\section{B. Business Framework of Sports Resource Information Platform Based on Big Data}

With the deepening of the concept of "Internet+", sports informationization has also been developed. Sports administrations and Sports Industry Management Co., Ltd. have basically constructed portals and APP applications. We should build platforms on this basis and make overall planning in advance to further clarify the platform business framework.

The business on sports resources information big data platform in accordance with the occurrence of the object can be divided into: management department-to-management department, management department-to-business and business-to-business users.

There are many sources of information on sports resources, which can be divided into internal systems of management departments and external systems of management departments. The internal systems of management departments include: track and field training center, stadium management committee, sports industry group Co., Ltd., stadiums, sports service guarantee center, etc.; external systems include: sports facilities of enterprises and institutions, fitness centers organized by social forces.

We shall build a sports resources information big data platform and tap existing sports resources data to provide support and services for various applications that require sports resource data.

\section{Construction of sports resources information big data platform}

Based on the sports resource information of relevant agencies such as the sports management department, enterprises and institutions and the fitness center held by the social forces, the big data platform of sports resources information, through the mining of data, provides support for the sports resources information applied in the management department, the relevant units and individuals based on sports resources information sharing mechanism.

The data platform shall comply with the construction standards and the Basic Requirements for Protection of Security Level of Information Systems (People's Republic of China National Standard GB/T 22239-2008) and shall be constructed by the infrastructure, big data system and big data application system.

Standard system: a scientific and feasible standard system is a key factor for the successful construction of a big data platform for sports resources information. We shall establish the "sports resources information big data platform expert committee", and the expert committee shall develop data platform technical standards, management standards and safety regulations.

Security: security system is the lifeline of the big data platform of sports resources information. We shall make full use of the technical features of big data platform to prevent illegal invasion and vulnerability attacks and strengthen the establishment of security clearance system with virus clearing and access setting as the core so as to ensure the data security and system security of the sports resources information big data platform.

Infrastructure: The construction of a big data platform for sports resources information requires the existing sports resources information of sports management departments, sports institutions and enterprises and fitness centers organized by social forces, and needs to collect real-time data from these departments. The stable, reliable and secure network channel, powerful hardware and software systems are the necessary conditions for the completion of data collection and integration. We should further increase the intensity of infrastructure construction, optimize the network system, upgrade and update the computer hardware and software equipment, and lay a solid material foundation for the big data platform.

Big Data System: Sports Resource Information Big Data System includes support data, data exchange and integration system, catalog management system, operation and maintenance system. The data exchange and integration system will be distributed in different business systems, diversified data collection, cleaning, exchange and integration and other operations. The processed data is then stored by the catalog management system.

Big Data Application: Big Data Application System is the core of big data platform of sports resource information and also the key to realize various applications. The platform can provide various types of big data services to sports management departments, enterprises and public institutions, fitness centers and individuals. For example, the sports administration department monitors the sports stadiums in real time; the user reserves the venue through the terminal according to the stadium distribution and the number of realtime users; and the user reserves the courses online according to the contents and time of the coach. 


\section{Design of sports resources information big data platform}

The sports resources information big data platform includes: support data, data exchange and integration system, catalog management system, operation and maintenance management system, interface system and platform system. The structure of sports resources information big data platform is shown as in Figure 1.

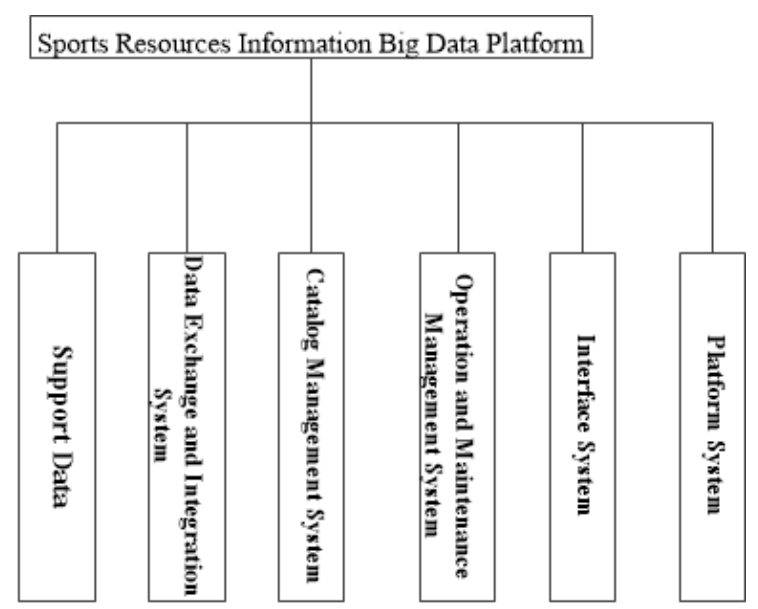

Figure 1. Structure of Sports Resources Information Big Data Platform

\section{1) Support data}

The support data can effectively connect sports resources information big data platform and sports resources database, and also can support the normal operation of other systems. It mainly contains metadata, exchange data, directory data, management data and security data.

The metadata, also known as the intermediary data, is the information about the organization of the data, the data fields and their relation. In short, the metadata is the data about the data; the exchange data is generated during the data exchange, which includes node data, parameter management Information, etc .; the directory data is mainly the directory information of sports information resource; the operation and other information generated by the operation and maintenance system collectively referred to as management data; the system failure frequency information and system security analysis data that record security situation of sports resources information big data platform becomes safety data.

\section{2) Data exchange and integration system}

The data exchange is based on a unified network and exchange protocol to achieve the exchange of information on sports resources between the relevant departments, avoid data chaos and repeat in the process of running exchange so as to achieve the synchronization of sports resources information and ensure data stability and security. The data exchange business model is shown in Figure 2.

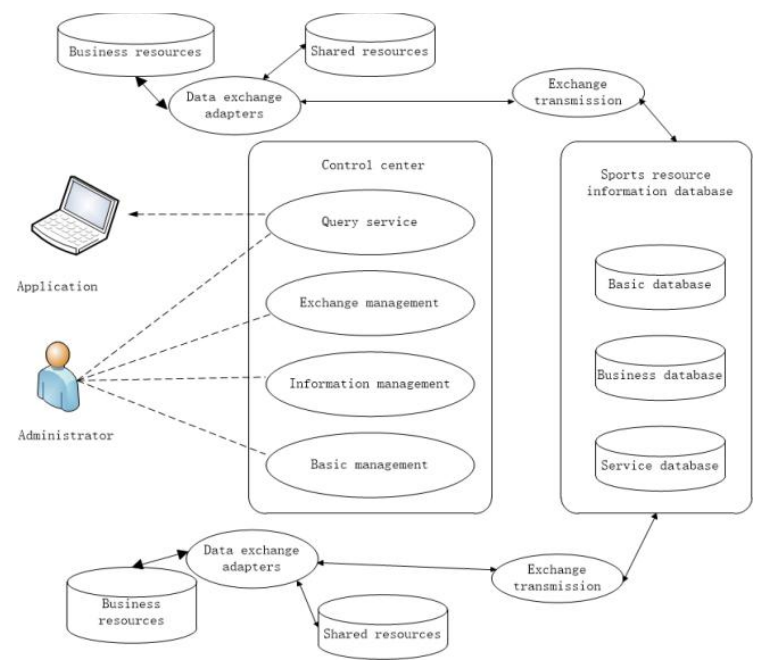

Figure 2. Data Exchange Business Model

The main function of data integration is collecting, cleaning, exchanging and integrating the sports resource information data dispersed in various departments, reanalyzing the data collection for relevance and causality and digging data deeply to form new and more valuable data resources. Data integration service system includes modules of integrated configuration, component management, process management and integration results information query. The integrated configuration module is used for dynamically configuring and integrating rules. The component management module is used to package data cleaning, conversion, alignment and splitting. The process management module is used for process management of various processes in the integration and implementation process; The integrated result information query module is used to provide query and statistics of the data integration results. The data integration business model is shown in Figure 3.

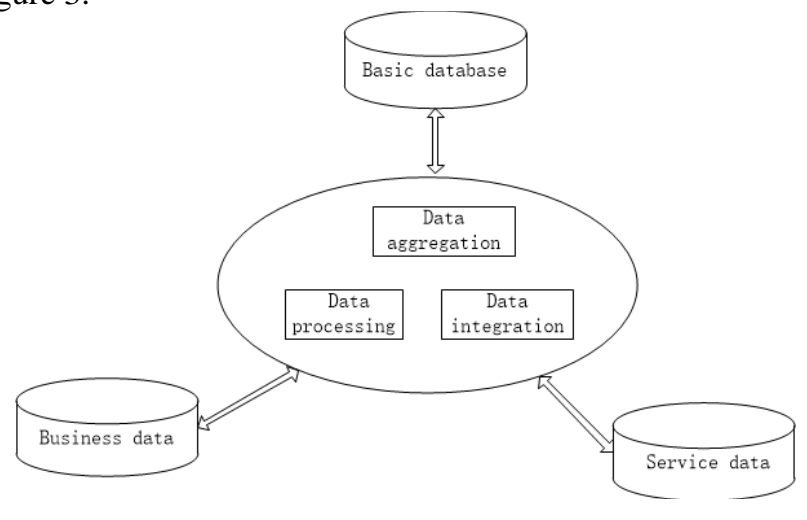

Figure 3. Data Exchange Business Model

\section{3) Catalog management system}

The catalog management system achieves its functionality through three subsystems like cataloging, transmission, management and service. Catalog system finishes metadata assignment and generates the contents of the directory in accordance with predetermined standards; 
directory transmission system completes the transmission of the directory contents between databases; directory management and service system realizes directory content audit, query, publishing and other functions.

4) Operation and maintenance management system

The O\&M Management System realizes the daily operation and maintenance and the security of Big Data Platform of Sports Resource Information. The main functions are as follows:

User Management: This function is mainly used to distinguish between the providers, managers and users of sports resource information, and users with different identities have different permissions; Resource Management: This function is mainly used for the management of metadata, with the query function; Application Management: This function is mainly used for reviewing and sorting out the distributed sports resource information, and charging the released information of paid sports resources; Security Maintenance and Management: realizing such functions as user identity authentication, data encryption and transmission, real-time data backup, and recording daily access status and fault information.

\section{5) Interface system}

The interface system is a channel for developers to call sports resource information. With this channel, developers can integrate the service of the platform with their own existing applications and directly develop the big data platform of sports resource information.

6) Platform system

The running window of sports resources information big data platform is the platform system. The platform system is user-oriented, providing support services according to different needs, which is used for a variety of platform-based applications in order to achieve the construction of sports resources information big data platform.

\section{PERFORMANCE EVALUATION OF SPORTS RESOURCES INFORMATION BIG DATA PLATFORM}

The objective evaluation of sports resources information big data platform is to master the use of sports resources information, improve the utilization of sports resources, further promote the development of sports informatization and improve the physical fitness of the people. The selection of performance evaluation indicators should be clear and easy to operate, combining qualitative indicators with quantitative indicators, establishing a performance evaluation index system that objectively reflects the big data platform of sports resources information, and scientifically evaluating the big data platform of sports resources information.

\section{CONCLUSION}

The Big Data Platform for Sports Resource Information covers such big data as investment in sports resources, costs, prices and benefits, involving sports management departments, enterprises and institutions, and fitness centers organized by social forces. Through the data cleaning, mining and other operations to sports resource information and based on the sports resource information sharing mechanism, the platform can provide the support of sports resource information applications. Problems such as unbalanced distribution of public sports resources, dislocation of supply and demand of sports resources and informatoin asymmetry of supply and demand of sports resources must be solved technically and the pace of sports information construction in China shall be accelerated to further enhance the national physique.

\section{ACKNOWLEDGMENT}

Subject: Routine Subject of Sports Bureau of Shaanxi Province in 2017: Big-Data-based Research on Integration and Application of Sports Resource in Shaanxi Province (Project No.17087);

Annual University-level Research Project of Xi'an Siyuan University in 2016: Planning and Research on Campus Card System(Project No.XASY-B1619).

\section{REFERENCES}

[1] [2] China State Council, Action Program for Big Data Development, [EB/OL] .http: //www.gov.cn/zhengce/content/201509/05/content_10137.htm, August 28, 2017. (references)

[3] Qu Haixu, Zhang Hujun and Cui Haiqing, Development and Application of Production and Operation Simulation System of Oil Company Based on Big Data [J].

[4] Zhang Jingbo, Research on the Improvement of Computer Network Reliability under the Background of "Internet+" $[\mathrm{J}]$.Journal of Liaoning Higher Vocational Technical Institute, vol.19 (06), pp.85$87,2017$.

[5] General Administration of Sport of China, National Fitness Program [EB/OL] .http //www.sport.gov.cn/n317/n10506/c730866/content.html, August 28, 2017.

[6] General Administration of Sport of China, "13th Five-Year Plan" for Sports Development, [EB/OL] .http: //news.xinhuanet.com/sports/2016-05/05/c_128960270.htm, August 28, 2017.

[7] Liu Liang and Wang Hui, Dispelling and Reforming Ways of Supply and Demand Contradictions of Public Sports Resources in China from the Perspective of Supply-side Reform [J]. Journal of Wuhan Institute of Physical Education, vol.50 (04), pp.53-55, 2016. 